Article

\title{
Perspectives on Constraining a Cosmological Constant-Type Parameter with Pulsar Timing in the Galactic Center
}

\section{Lorenzo Iorio}

Ministero dell'Istruzione, dell'Università e della Ricerca (M.I.U.R.)-Istruzione, Viale Unità di Italia 68, 70125 Bari (BA), Italy; lorenzo.iorio@libero.it

Received: 19 February 2018; Accepted: 19 March 2018; Published: 26 March 2018

\begin{abstract}
Independent tests aiming to constrain the value of the cosmological constant $\Lambda$ are usually difficult because of its extreme smallness $\left(\Lambda \simeq 1 \times 10^{-52} \mathrm{~m}^{-2}\right.$, or $2.89 \times 10^{-122}$ in Planck units $)$. Bounds on it from Solar System orbital motions determined with spacecraft tracking are currently at the $\simeq 10^{-43}-10^{-44} \mathrm{~m}^{-2}\left(5-1 \times 10^{-113}\right.$ in Planck units $)$ level, but they may turn out to be optimistic since $\Lambda$ has not yet been explicitly modeled in the planetary data reductions. Accurate $\left(\sigma_{\tau_{\mathrm{p}}} \simeq 1-10 \mu \mathrm{s}\right)$ timing of expected pulsars orbiting the Black Hole at the Galactic Center, preferably along highly eccentric and wide orbits, might, at least in principle, improve the planetary constraints by several orders of magnitude. By looking at the average time shift per orbit $\overline{\Delta \delta}_{\mathrm{p}}^{\Lambda}$, an S2-like orbital configuration with $e=0.8839, P_{\mathrm{b}}=16 \mathrm{yr}$ would permit a preliminarily upper bound of the order of $|\Lambda| \lesssim 9 \times 10^{-47} \mathrm{~m}^{-2}\left(\lesssim 2 \times 10^{-116}\right.$ in Planck units $)$ if only $\sigma_{\tau_{\mathrm{p}}}$ were to be considered. Our results can be easily extended to modified models of gravity using $\Lambda$-type parameters.
\end{abstract}

Keywords: astrophysical studies of gravity; general relativity; cosmological constant; neutron stars \& pulsars; classical black holes

\section{Introduction}

The cosmological constant (CC) $\Lambda$ [1-8] is the easiest way to explain certain large-scale features of the universe like the acceleration of its expansion $[9,10]$ and the growth of fluctuations by gravity [11] within General Relativity (GR) assumed as a fundamental ingredient of the standard $\Lambda$ CDM model [12]; for a recent overview of the status and future challenges of the Einsteinian theory of gravitation, see, e.g., Debono and Smoot [13]. Interestingly, the CC was considered before Einstein for the possible modification of the Poisson equation in the framework of the Newtonian gravity [14]. The CC can be expressed in terms of the Hubble parameter $H_{0}$ and the ratio $\Omega_{\Lambda}$ between the density due to the cosmological constant itself $\varrho_{\Lambda}=(1 / 8 \pi) c^{2} \Lambda G^{-1}$ and the critical density $\varrho_{\text {crit }}=(3 / 8 \pi) H_{0}^{2} G^{-1}$ as $\Lambda=3 H_{0}^{2} \Omega_{\Lambda} c^{-2}$, where [15] $H_{0}=67.74 \pm 0.46 \mathrm{~km} \mathrm{~s}^{-1} \mathrm{Mpc}^{-1}, \Omega_{\Lambda}=0.6911 \pm 0.0062$. As such, its most recent value inferable from the measurements of the Cosmic Microwave Background (CMB) power spectra by the satellite Planck reads

$$
\Lambda=(1.11 \pm 0.02) \times 10^{-52} \mathrm{~m}^{-2}
$$

In order to relate it to possible symmetry breaking in gravity [16], the CC is sometimes written as a very tiny dimensionless parameter essentially by multiplying it by the square of the Planck length $\ell_{\mathrm{P}}=\sqrt{\hbar G c^{-3}}=1.61 \times 10^{-35} \mathrm{~m}$. Thus, one gets, in Planck units,

$$
\Lambda=2.89 \times 10^{-122} .
$$


A CC-type parameterization occurs also in several classes of long range modified models of gravity aiming to explain, in a unified way, seemingly distinct features of the cosmic dynamics like inflation, late-time acceleration and even dark matter [17-28].

Ever since the time of Einstein, who, on the backdrop of what is mathematically feasible with the Poisson equation, included $\Lambda$ in his GR field equations to obtain a non-expanding, static cosmological model [29], the introduction of the CC has always been justified from an observational/experimental point of view by arguing that it would not be in contrast with any observed effects in local systems like, e.g., orbital motions in gravitationally bound binary systems because of its extreme smallness. As a consequence, there are not yet independent, non-cosmological tests of the CC itself for which only relatively loose constraints from planetary motions of the Solar System exist in the literature. So far, most of the investigations on the consequences of the CC in local binary systems have focused on the anomalous pericenter precession induced by $\Lambda$ [30-49] on the basis of a Hooke-type perturbing potential [32,33]

$$
U_{\Lambda}=-\frac{1}{6} \Lambda c^{2} r^{2}
$$

arising in the framework of the Schwarzschild-de Sitter spacetime [32,50,51]. Equation (3) yields the radial extra-acceleration $[32,33]$

$$
A_{\Lambda}=\frac{1}{3} \Lambda c^{2} \mathbf{r} .
$$

The latest upper limits on the absolute value of $\Lambda$, inferred within the framework of $f(T)$ gravity from the anomalous perihelion precessions of some of the planets of the Solar System tightly constrained with the INPOP10a ephemerides [52], are of the order of [47]

$$
|\Lambda| \lesssim 2 \times 10^{-43} \mathrm{~m}^{-2},
$$

corresponding to

$$
|\Lambda| \lesssim 5 \times 10^{-113},
$$

in Planck units.The Earth-Saturn range residuals constructed from the telemetry of the Cassini spacecraft [53] yielded an upper limit of the order of [48]

$$
|\Lambda| \lesssim 5 \times 10^{-44} \mathrm{~m}^{-2},
$$

i.e.,

$$
|\Lambda| \lesssim 1 \times 10^{-113},
$$

in Planck units. Iorio et al. [54] suggested that a challenging analysis of the telemetry of the New Horizons spacecraft might improve the limit of Equation (7) by about one order of magnitude. On the other hand, the bounds of Equations (5)-(7) may be somehow optimistic since they were inferred without explicitly modeling Equation (4) in the dynamical force models of the ephemerides. As such, its signature may have been removed from the post-fit residuals to a certain extent, being partially absorbed in the estimation of, for example, the planets' initial state vectors. Such a possibility was investigated by simulating observations of major bodies of the Solar System in the case of some modified models of gravity [55]. Thus, more realistic constraints might yield larger values for the allowed upper bound on $\Lambda$.

In this paper, we will show that the future, long waited discovery of pulsars revolving around the putative Supermassive Black Hole (SMBH) in the Galactic Center (GC) at Sgr A* [56-59] along sufficiently wide and eccentric orbits and their timing accurate to the $\sigma_{\tau_{\mathrm{p}}} \simeq 1-10 \mu \mathrm{s}$ level $[60,61]$, might allow, in principle, substantial improvement on the planetary bounds of Equations (5)-(7) by several orders of magnitude, getting, perhaps, closer to the level of Equation (1) itself under certain fortunate conditions. The possibility that traveling gravitational waves can be used in a foreseeable future for local measurements of the CC through their impact on Pulsar Timing Arrays (PTA) is discussed in 
Espriu [62]. In Section 2 we will analytically work out the perturbation $\Delta \delta \tau_{\mathrm{p}}^{\Lambda}$ induced by $\Lambda$ on the pulsar's timing periodic variation $\delta \tau_{\mathrm{p}}$ due to its orbital motion around the $\mathrm{SMBH}$; we will follow the approach put forth in Iorio [63] applying it to Equation (4). We will neglect the time shifts due to the CC on the propagation of the electromagnetic waves [64]. Despite it can be shown that, for certain values of the initial conditions, an extremely wide orbital configuration like, say, that of the actually existing star S85 may yield values of the instantaneous changes $\Delta \delta \tau_{\mathfrak{p}}^{\Lambda}(t)$ as large as just $\simeq 1-10 \mu \mathrm{s}$, caution is in order because of, for example, the very likely systematic bias induced on such an extended orbit by the poorly known mass background in the GC [65-68]. Also, the accurate knowledge of the SMBH physical parameters like mass, angular momentum and quadrupole moment would be of crucial importance because of the competing $\mathrm{pN}$ orbital timing signatures $\Delta \delta \tau_{\mathrm{p}}^{\mathrm{pN}}$, which would superimpose to the CC effect. Finally, also the orbital parameters of the pulsar should be determined over a relatively short time interval $\Delta T$ with respect to its extremely long orbital period $P_{\mathrm{b}}$. If, instead, a closer pulsar is considered, it makes sense to look at its net orbital time shift per orbit $\overline{\Delta \delta}_{\mathrm{p}}^{\Lambda}$. Zhang and Saha [69] recently investigated the possibility of constraining the SMBH's spin with such kind of rapidly orbiting pulsars. See also De Laurentis et al. [70]. In Section 3, it will be shown that a S2-type orbital geometry, summarized in Table A1, would allow, in principle, improvement to the planetary bounds of Equations (5)-(7) by about 3-4 orders of magnitude. A strategy to overcome the potentially serious bias posed by the competing post-Newtonian $(\mathrm{pN})$ orbital time delays driven by the SMBHS's mass, spin and quadrupole moment will be discussed as well. In Section 4, we summarize our findings and offer our conclusions.

\section{Calculating the Perturbation of the Orbital Component of the Time Shift Due to the Cosmological Constant}

Here, the analytical method devised in Iorio [63], relying upon Casotto [71], will be applied to the perturbing acceleration of Equation (4) with some technical modifications. Indeed, since, in this case, the use of the eccentric anomaly $E$ as a fast variable of integration instead of the true anomaly $f$ turns out to be computationally more convenient, Equations (30) and (31) of Casotto [71], giving the radial and transverse components of the perturbation $\Delta \mathbf{r}$ of the position vector $\mathbf{r}$ and used in Iorio [63] as Equations (3) and (4), have to be replaced with Equations (36) and (37) of Casotto [71], i.e.,

$$
\begin{aligned}
& \Delta \mathrm{r}_{\rho}(E)=\frac{r(E)}{a} \Delta a(E)-\frac{r(E)(e+\cos f)}{1-e^{2}} \Delta e(E)+\frac{r(E) e \sin f}{\sqrt{1-e^{2}}} \Delta E(E), \\
& \Delta \mathrm{r}_{\sigma}(E)=\frac{r(E) \sin f}{1-e^{2}} \Delta e(E)+a \sqrt{1-e^{2}} \Delta E(E)+r(E)[\cos I \Delta \Omega(E)+\Delta \omega(E)] .
\end{aligned}
$$

Equation (32) of Casotto [71], giving the out-of-plane component $\Delta \mathrm{r}_{v}$ of the perturbation $\Delta \mathbf{r}$ of the position vector $\mathbf{r}$ and used in Iorio [63] as Equation (5), remains unchanged. Thus, the perturbation of the $z$ component of the pulsar's position vector $\mathbf{r}$ reads

$$
\begin{aligned}
\Delta \mathrm{r}_{z} & =\frac{r(E)}{a} \sin I \sin u \Delta a(E)-\frac{r(E) \sin I(\sin \omega+e \sin u)}{1-e^{2}} \Delta e(E)+r(E) \cos I \sin u \Delta I(E)+ \\
& +r(E) \sin I \cos u \Delta \omega(E)+\frac{\sin I\left[a\left(1-e^{2}\right) \cos u+e r(E) \sin f \sin u\right]}{\sqrt{1-e^{2}}} \Delta E(E) .
\end{aligned}
$$

From Iorio [63], it is $\Delta \delta \tau_{\mathrm{p}}=\Delta \mathrm{r}_{z} c^{-1}$ in a coordinate system whose reference $z$ axis points towards the observer perpendicularly to the plane of the sky spanned by the reference $\{x, y\}$ plane. In Equations (9)-(11), the instantaneous shift $\Delta E(E)$ of the eccentric anomaly can be expressed, in turn, in terms of the perturbations $\Delta \mathcal{M}(E), \Delta e(E)$ of the mean anomaly and the eccentricity, respectively, according to Equation (A.5) of Casotto [71], i.e., 


$$
\Delta E(E)=\frac{a}{r(E)}[\Delta \mathcal{M}(E)+\sin E \Delta e(E)] .
$$

The instantaneous shifts of the osculating orbital elements are to be computed in terms of $E$ as

$$
\Delta \kappa(E)=\int_{E_{0}}^{E} \frac{\mathrm{d} \kappa}{\mathrm{d} t} \frac{\mathrm{d} t}{\mathrm{~d} E^{\prime}} d E^{\prime}, \kappa=a, e, I, \Omega, \omega ;
$$

with the aid of the standard formulas of celestial mechanics

$$
\begin{aligned}
\sin f & =\frac{\sqrt{1-e^{2}} \sin E}{1-e \cos E}, \\
\cos f & =\frac{\cos E-e}{1-e \cos E}, \\
r(E) & =a(1-e \cos E), \\
\frac{\mathrm{d} t}{\mathrm{~d} E} & =\frac{1-e \cos E}{n_{\mathrm{b}}},
\end{aligned}
$$

applied to the usual Gauss equations for the variation of the elements yielding $d \kappa / d t$. The calculation of the perturbation $\Delta \mathcal{M}(E)$ of the mean anomaly has to be performed as shown in Iorio [63], whose Equations (20) and (21) are to be calculated with $E$. The CC-induced instantaneous perturbations of the osculating orbital elements turn out to be

$$
\begin{aligned}
\Delta a(E) & =\frac{c^{2} \Lambda a e\left(\cos E-\cos E_{0}\right)\left[-2+e\left(\cos E+\cos E_{0}\right)\right]}{3 n_{\mathrm{b}}^{2}} \\
\Delta e(E) & =\frac{c^{2} \Lambda\left(1-e^{2}\right)\left(\cos E-\cos E_{0}\right)\left[-2+e\left(\cos E+\cos E_{0}\right)\right]}{6 n_{\mathrm{b}}^{2}} \\
\Delta I(E) & =0 \\
\Delta \Omega(E) & =0 \\
\Delta \omega(E) & =\frac{c^{2} \Lambda \sqrt{1-e^{2}}}{12 e n_{\mathrm{b}}^{2}}\left[4\left(1+e^{2}\right) \sin E_{0}-e\left(6 E_{0}-6 E+\sin 2 E_{0}\right)-\right. \\
& \left.-4\left(1+e^{2}\right) \sin E+e \sin 2 E\right] \\
\Delta \mathcal{M}(E) & =\frac{c^{2} \Lambda}{72 e n_{\mathrm{b}}^{2}}\left\{12 e\left(7+6 e^{2}\right)\left(E_{0}-E\right)-4\left(6+54 e^{2}+7 e^{4}\right) \sin E_{0}+6 e \sin 2 E_{0}+\right. \\
& +3\left(8+72 e^{2}+7 e^{4}\right) \sin E+2 e^{3}\left[9\left(E-E_{0}\right)+e\left(2 \sin E_{0}-9 \sin E\right)\right] \cos 2 E_{0}+ \\
& +6 e^{2}\left[7 e \sin E_{0}+12\left(E_{0}-E+e \sin E\right)\right] \cos E_{0}- \\
& \left.-3 e\left(2+19 e^{2}\right) \sin 2 E+7 e^{4} \sin 3 E\right\} .
\end{aligned}
$$


By inserting Equations (19) and (23) in Equation (12), it is possible to explicitly infer the instantaneous perturbation of the eccentric anomaly

$$
\begin{aligned}
\Delta E(E) & =-\frac{c^{2} \Lambda}{72 e n_{\mathrm{b}}^{2}(1-e \cos E)}\left\{12\left[e\left(7+6 e^{2}\right)\left(E-E_{0}\right)+2\left(\sin E_{0}-\sin E\right)\right]+\right. \\
& +e\left[6 e\left(36+5 e^{2}\right) \sin E_{0}-3\left(2+7 e^{2}\right) \sin 2 E_{0}-2 e^{3} \sin 3 E_{0}-3 e\left(71+8 e^{2}\right) \sin E+\right. \\
& +\left(3 e E_{0}-3 e E+\sin E+2 e^{2} \sin E\right)\left(6 e \cos 2 E_{0}-24 \cos E_{0}\right)+ \\
& \left.\left.+9\left(2+5 e^{2}\right) \sin 2 E-e\left(3+4 e^{2}\right) \sin 3 E\right]\right\} .
\end{aligned}
$$

By inserting Equations (18)-(22) and Equation (24) in Equation (11) and using Equations (14)-(16) allows one to obtain the instantaneous perturbation $\Delta \delta \tau_{\mathrm{p}}^{\Lambda}(E)$ of the orbital time shift of the pulsar $\mathrm{p}$ due to $\Lambda$. It is

$$
\Delta \delta \tau_{\mathrm{p}}^{\Lambda}(E)=\frac{c \Lambda a \sin I}{72 n_{\mathrm{b}}^{2}} \mathcal{L}\left(E ; E_{0}, e, \omega\right),
$$

where $\mathcal{L}\left(E ; E_{0}, e, \omega\right)$ is a function of $E$ and the parameters $E_{0}, e, \omega$ definitely too cumbersome to be explicitly displayed. Thus, we show only the leading term of Equation (25);

$$
\begin{aligned}
\Delta \delta \tau_{\mathrm{p}}^{\Lambda}(E) & \simeq \frac{c \Lambda a \sin I}{6 n_{\mathrm{b}}^{2}}\left[4\left(E_{0}-E\right) \cos (E+\omega)-\sin \left(E_{0}-2 E-\omega\right)-\right. \\
& \left.-3 \sin \left(E_{0}+\omega\right)+2 \sin (E+\omega)\right]+\mathcal{O}\left(e^{k}\right), k \geq 1
\end{aligned}
$$

It is important to note from Equation (25) that $\Delta \delta \tau_{\mathrm{p}}^{\Lambda}$ is proportional to the fourth power of the semimajor axis $a$, which characterizes the size of the pulsar's orbit, and is inversely proportional to the mass of the SMBH.

The net shift per orbit can be calculated from Equation (25) with $E \rightarrow E_{0}+2 \pi$ : it turns out to be

$$
\begin{aligned}
{\overline{\Delta \delta \tau_{\mathrm{p}}}}^{\Lambda} & =-\frac{\pi c \Lambda a \sin I}{12 n_{\mathrm{b}}^{2}} \frac{1}{\left(1-e \cos E_{0}\right)}\left\{\sqrt { 1 - e ^ { 2 } } \left[\left(16+9 e^{2}\right) \cos E_{0}+\right.\right. \\
& \left.+3 e\left(10+6 \cos 2 E_{0}-e \cos 3 E_{0}\right)\right] \cos \omega-16 \sin E_{0} \sin \omega+ \\
& \left.+6 e\left[2\left(-3+e^{2}\right) \cos E_{0}+e\left(-6+\cos 2 E_{0}\right)\right] \sin E_{0} \sin \omega\right\}
\end{aligned}
$$

It can be noted that also Equation (27) depends on the initial conditions through $E_{0}$. It is also important to stress that both Equations (25) and (27) were worked out without any a priori simplifying approximations about the pulsar's orbital configuration; they hold for all values of $e$. It is a key feature in view of the highly eccentric orbits revealed so far in the GC.

\section{The Opportunity Offered by Hypotetical Pulsars in the Galactic Center}

Let us now move to the compact object located in Sgr A*. For an interesting multidisciplinary discussion about the possibility that it is, actually, a SMBH or something else, see the recent overview in Eckart et al. [72]. However, our results will be unaffected by the alternative possibilities discussed there since their spacetimes are indistinguishable from that of a SMBH for the pulsars' orbital motions of interest here. 
In order to explore the opportunity offered by our results to effectively constrain the CC with pulsar timing in the GC, let us consider a putative pulsar whose orbital period $P_{\mathrm{b}}$ is short enough to allow to monitor at least one full revolution during a timing campaign. In this case, by suitably choosing the initial orbital phase $E_{0}$, it would be possible to profitably use Equation (27) in order to maximize it; indeed, in principle, Equation (27) can even vanish. To this aim, for the sake of concreteness, let us assume a S2-type orbital configuration characterized by $P_{\mathrm{b}}=16 \mathrm{yr}, e=0.8839$ [73]. It turns out that the maximum of the absolute value of Equation (27) occurs for $E_{0}=342.08 \mathrm{deg}$, which corresponds to almost an orbital period after the time of periastron passage, yielding an upper bound on the CC as little as

$$
|\Lambda| \lesssim 9 \times 10^{-47} \mathrm{~m}^{-2}\left(\lesssim 2 \times 10^{-116} \text { in Planck units }\right)
$$

for a timing accuracy of $\sigma_{\tau_{\mathrm{p}}} \simeq 1 \mu \mathrm{s}$. It should be noted that Equation (28) is 3-4 orders magnitude better than the (likely optimistic) planetary bounds of Equations (5)-(7). Figure A1 depicts the plot of Equation (27) as a function of $E_{0}$. If we modify some of the parameters of the pulsar's orbital configuration by adopting, say, $P_{\mathrm{b}}=30 \mathrm{yr}, e=0.987, I=90 \mathrm{deg}$, it is possible to improve the bound on the $\mathrm{CC}$ to the level

$$
|\Lambda| \lesssim 4 \times 10^{-48} \mathrm{~m}^{-2}\left(\lesssim 1 \times 10^{-117} \text { in Planck units }\right)
$$

for $E_{0}=354.04 \mathrm{deg}$. About the figures in Equations (28)-(29), inferred by considering only $\sigma_{\tau_{\mathrm{p}}}$ as source of observational error, it must be stressed that they should be regarded with caution as preliminary and just indicative of the potential of the approach proposed. If not explicitly modeled and simultaneously estimated in actual pulsar timing data reductions, the CC-induced signature may be partially removed from the resulting residual. As such, the resulting bounds may be weaker than those in Equations (28)-(29). Further dedicated analyses should be made by simulating observations and fitting a full orbital model to them in order to assess how good the input values are recovered. A possible source of systematic uncertainty is represented by the mismodelled part of the competing averaged orbital time shifts induced by the standard post-Newtonian $(\mathrm{pN})$ effects due to the current experimental errors in the SMBH's parameters entering their formulas. For example, according to Equation (35) of Iorio [63], the amplitude of the $1 \mathrm{pN}$ gravitoelectric average time shift ${\overline{\Delta \delta \tau_{\mathrm{p}}^{\mathrm{GE}}}}_{\text {is }}$ proportional to $\mu_{\bullet} c^{-3}=22 \mathrm{~s}$, while the mass of the $\mathrm{SMBH}$ is currently known at a $\simeq 7 \%$ level of accuracy [73]. Analogous considerations hold for the Lense-Thirring (Equation (51) of Iorio [63]) and quadrupole (Equation (83) of Iorio [63]) average shifts. In principle, such an issue could be circumvented if $N$ pulsars $j$ with different orbital configurations will be discovered. Indeed, in this case, it could be possible to write down for each of them an analytical expression

$$
\overline{\Delta \delta \tau}_{j}^{\exp }=\overline{\Delta \delta \tau}_{j}^{\mathrm{GE}}+\overline{\Delta \delta \tau}_{j}^{\mathrm{LT}}+\overline{\Delta \delta \tau}_{j}^{\mathrm{Q}_{2}}+\overline{\Delta \delta \tau}_{j}^{\Lambda}, j=1,2, \ldots N
$$

for their measured average orbital time shift $\overline{\Delta \delta}_{j}^{\exp }$ as a sum of the $\mathrm{pN}$ terms plus the CC one by treating $\mu_{\bullet}, S_{\bullet}, Q_{2}^{\bullet}, \Lambda$, which enter each term of Equation (30) as multiplicative scaling parameters, as unknowns of the resulting linear system of algebraic equations. Solving for them, it would be possible to obtain, among other things, an expression for $\Lambda$ independent, by construction, of the mismodeled SMBH's physical parameters. Such an approach could be extended also to other dynamical effects impacting the pulsar's average orbital time shift like, e.g., third-body perturbations.

Recently, the upper bound

$$
\left|\dot{\omega}_{\mathrm{S} 2}\right| \lesssim 1.6 \times 10^{-3} \mathrm{yr}^{-1}=9.2 \mathrm{deg} \mathrm{cty}^{-1}
$$

on the periastron precession of the real star S2 was inferred in Hees et al. [74]. By combining Equation (31) with the well known analytical expression for the $\Lambda$-induced pericenter precession (see the references cited in Section 1) 


$$
\dot{\omega}_{\Lambda}=\frac{1}{2}\left(\frac{\Lambda c^{2}}{n_{\mathrm{b}}}\right) \sqrt{1-e^{2}},
$$

it is possible to infer a tentative upper limit on the CC of the order of

$$
|\Lambda| \lesssim 3 \times 10^{-35} \mathrm{~m}^{2}\left(\lesssim 8 \times 10^{-105} \text { in Planck units }\right)
$$

For much more distant pulsars, major sources of systematic uncertainty would be given by the still poorly mass background and the difficulty of effectively constraining the parameters of extremely wide orbits [75] and of the Black Hole itself over a relatively short observational time interval $\Delta T$ with respect to the expected extremely long orbital period $P_{\mathrm{b}}$ of the neutron star.

\section{Summary and Conclusions}

In this paper, we analytically calculated the perturbation $\Delta \delta \tau_{\mathrm{p}}^{\Lambda}$ induced by the $\mathrm{CC} \Lambda$ on the orbital part of the time variation $\delta \tau_{\mathrm{p}}$ of a hypothetical pulsar p orbiting the SMBH in Sgr A*. We did not restrict to any particular orbital configuration, and our results are, thus, exact with respect to the eccentricity $e$; it is an important feature since most of the main sequence stars discovered so far in the GC move along highly eccentric orbits. We obtained both the instantaneous change $\Delta \delta \tau_{\mathrm{p}}^{\Lambda}(E)$ and the net shift per orbit $\overline{\Delta \delta \tau}_{\mathrm{p}}^{\Lambda}$ : they are proportional to $c \Lambda a^{4} \sin I \mu_{\bullet}^{-1}$. A distinctive feature of both of them is their explicit dependence on the initial value $E_{0}$ of the orbital phase. Our results hold also for a wide class of long-range modified models of gravity generating an extra-potential quadratic in the distance $r$.

We applied our results to some putative scenarios by adopting, for the sake of definiteness, the orbital configurations of one actually existing main sequence star orbiting Sgr $\mathrm{A}^{*}$. By considering a S2-type orbit with $P_{\mathrm{b}}=16 \mathrm{yr}$, it is meaningful to look at the averaged time shift $\overline{\Delta \delta}_{\mathrm{p}}^{\Lambda}$. It turns out that, for a careful choice of the initial orbital phase $E_{0}$, it would be possible, in principle, to infer an upper bound $|\Lambda| \lesssim 9 \times 10^{-47} \mathrm{~m}^{-2}$, corresponding to $\lesssim 2 \times 10^{-116}$ in Planck units, by assuming a pulsar timing accuracy of $\sigma_{\tau_{\mathrm{p}}} \simeq 1 \mu \mathrm{s}$. It would be $3-4$ orders of magnitude better than the current, likely optimistic, constraints from Solar System's planetary orbital motions. On the other hand, it should be stressed that the very same aforementioned bound on $\Lambda$, derived by accounting for only $\sigma_{\tau_{p}}$, may be optimistic in view of possible partial removal of the sought signature if not explicitly modeled and solved for in actual data reductions. As a suggestion for further dedicated investigations, simulating the observations and fitting a complete dynamical orbital model to them would be needed in order to assess how accurately the input values can be recovered. The bias due to the errors in the physical parameters of the SMBH entering the competing $\mathrm{pN}$ net shifts per orbit could be eliminated by setting up suitably designed linear combinations of the time delays measured for several pulsars. In the case of much more distant pulsars, using the orbital averaged time shift $\overline{\Delta \delta}_{\mathrm{p}}^{\Lambda}$ is unfeasible; only instantaneous values $\Delta \delta \tau_{\mathrm{p}}^{\Lambda}(E)$ could be, in principle, measured. On the other hand, too wide and slow orbits may be impacted by the still poorly known mass background in the GC, and it would be difficult to effectively constrain the pulsar's orbital parameters over a relatively short time interval with respect to its extremely long orbital period.

Acknowledgments: I would like to thank two attentive referees for their precious critical remarks.

Conflicts of Interest: The author declares no conflict of interest. 


\section{Appendix A}

\section{Appendix A.1. Notations and definitions}

Some basic notations and definitions used in the text are listed below [76-79]. In the case treated in this paper, the unseen companion c of the pulsar $\mathrm{p}$ is the SMBH of mass $M_{\bullet}$, so that $m_{\mathrm{c}}=M_{\bullet} \gg m_{\mathrm{p}}$ and $a_{\mathrm{p}} \simeq a$.

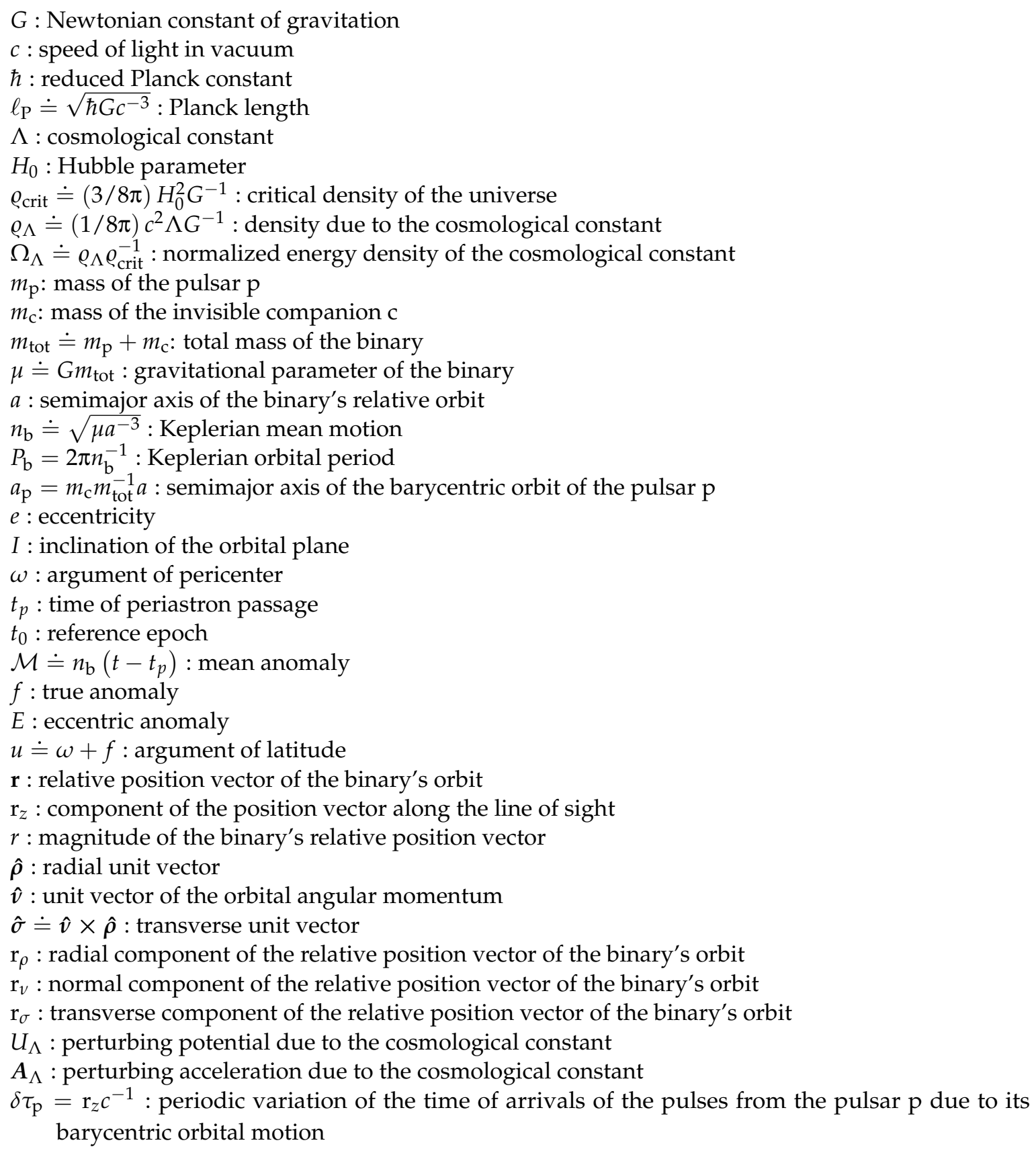


Appendix A.2. Tables and Figures

Table A1. Relevant physical and orbital parameters of the S2 star and the SMBH at the GC along with their estimated uncertainties according to Table 3 of Gillessen et al. [73]; they are referred to the epoch 2000.0. $D_{0}$ is the distance to Sgr $\mathrm{A}^{*}$. The linear size of the semimajor axis of S2 is $a=1044$ au.

\begin{tabular}{ll}
\hline Estimated Parameter & Value \\
\hline$M_{\bullet}$ & $4.28 \pm\left. 0.10\right|_{\text {stat }} \pm\left. 0.21\right|_{\text {sys }} \times 10^{6} \mathrm{M} \odot$ \\
$D_{0}$ & $8.32 \pm\left. 0.07\right|_{\text {stat }} \pm\left. 0.14\right|_{\text {sys }} \mathrm{kpc}$ \\
$P_{\mathrm{b}}$ & $16.00 \pm 0.02 \mathrm{yr}$ \\
$a$ & $0.1255 \pm 0.0009 \mathrm{arcsec}$ \\
$e$ & $0.8839 \pm 0.0019$ \\
$I$ & $134.18 \pm 0.40 \mathrm{deg}$ \\
$\Omega$ & $226.94 \pm 0.60 \mathrm{deg}$ \\
$\omega$ & $65.51 \pm 0.57 \mathrm{deg}$ \\
$t_{p}$ & $2002.33 \pm 0.01$ calendar year \\
\hline
\end{tabular}

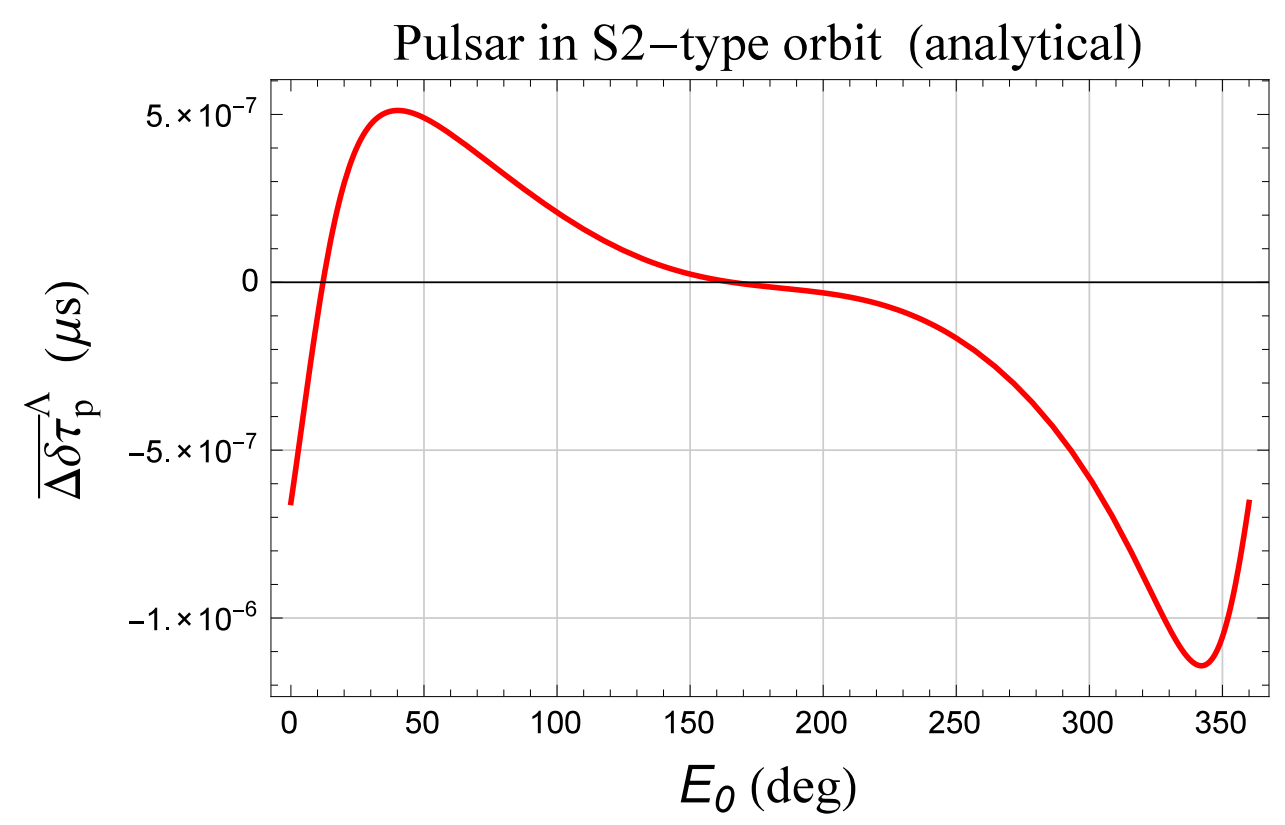

Figure A1. Average orbital time shift per orbit $\overline{\Delta \delta \tau}_{\mathrm{p}}^{\Lambda}$, in $\mu$ s, of a hypothetical pulsar in Sgr A* obtained analytically from Equation (27) along with the value of Equation (1) for $\Lambda$ as a function of the initial phase $E_{0}$. The orbital configuration of the S2 star, quoted in Table A1, was adopted. It can be noted that $\overline{\Delta \delta \tau}_{\mathrm{p}}^{\Lambda}$ vanishes for two given values of $E_{0}$; the largest absolute value occurs for $E_{0}=342.08 \mathrm{deg}$. By assuming a pulsar timing accuracy of $\sigma_{\tau_{\mathrm{p}}}=1 \mu \mathrm{s}$, it translates to an upper bound on $\Lambda$ of the order of $|\Lambda| \leq 9 \times 10^{-47} \mathrm{~m}^{-2}\left(\lesssim 10^{-} 116\right.$ in Planck units).

\section{References}

1. Weinberg, S. The cosmological constant problem. Rev. Mod. Phys. 1989, 61, 1-23.

2. Carroll, S.M.; Press, W.H.; Turner, E.L. The cosmological constant. Annu. Rev. Astron. Astrophys. 1992, 30, 499-542.

3. Carroll, S.M. The Cosmological Constant. Living Rev. Relativ. 2001, 4, 1.

4. Peebles, P.J.; Ratra, B. The cosmological constant and dark energy. Rev. Mod. Phys. 2003, 75, 559-606.

5. Padmanabhan, T. Cosmological constant-the weight of the vacuum. Phys. Rep. 2003, 380, 235-320.

6. Carroll, S.M. Spacetime and Geometry. An Introduction to General Relativity; Addison Wesley: San Francisco, CA, USA, 2004. 
7. Davis, T.; Griffen, B. Cosmological constant. Scholarpedia 2010, 5, 4473.

8. O'Raifeartaigh, C.; O'Keeffe, M.; Nahm, W.; Mitton, S. One Hundred Years of the Cosmological Constant: from 'Superfluous Stunt' to Dark Energy, Eur. Phys. J. H 2018, 43, 1-45.

9. Riess, A.G.; Filippenko, A.V.; Challis, P.; Clocchiatti, A.; Diercks, A.; Garnavich, P.M.; Gilliland, R.L.; Hogan, C.J.; Jha, S.; Kirshner, R.P.; et al. Observational Evidence from Supernovae for an Accelerating Universe and a Cosmological Constant. Astron. J. 1998, 116, 1009-1038.

10. Perlmutter, S.; Aldering, G.; Goldhaber, G.; Knop, R.A.; Nugent, P.; Castro, P.G.; Deustua, S.; Fabbro, S.; Goobar, A.; Groom, D.E.; et al. Measurements of $\Omega$ and $\Lambda$ from 42 High-Redshift Supernovae. Astrophys. J. 1999, 517, 565-586.

11. Nesseris, S.; Perivolaropoulos, L. Testing $\Lambda \mathrm{CDM}$ with the growth function $\delta(\mathrm{a})$ : Current constraints. Phys. Rev. D 2008, 77, 023504.

12. Spergel, D.N. The dark side of cosmology: Dark matter and dark energy. Science 2015, 347, 1100-1102.

13. Debono, I.; Smoot, G.F. General Relativity and Cosmology: Unsolved Questions and Future Directions. Universe 2016, 2, 23.

14. Seeliger, H. Über das Newton'sche Gravitationsgesetz. Astron. Nachr. 1895, 137, 129-136.

15. Ade, P.A.; Aghanim, N.; Arnaud, M.; Ashdown, M.; Aumont, J.; Baccigalupi, C.; Banday, A.J.; Barreiro, R.B.; Bartlett, J.G.; Bartolo, N.; et al. [Planck Collaboration] Planck 2015 results. XIII. Cosmological parameters. Astron. Astrophys. 2016, 594, A13.

16. Mielke, E.W. Weak equivalence principle from a spontaneously broken gauge theory of gravity. Phys. Lett. B 2011, 702, 187-190.

17. Nojiri, S.; Odintsov, S.D. Introduction to modified gravity and gravitational alternative for dark energy. Int. J. Geom. Methods Mod. Phys. 2007, 4, 115-146.

18. Nojiri, S.; Odintsov, S.D. Modified gravity as an alternative for $\Lambda$ CDM cosmology. J. Phys. A Math. Gen. 2007, 40, 6725-6732.

19. Dunsby, P.K.S.; Elizalde, E.; Goswami, R.; Odintsov, S.; Saez-Gomez, D. $\Lambda$ CDM universe in f(R) gravity. Phys. Rev. D 2010, 82, 023519.

20. De Felice, A.; Tsujikawa, S. f(R) Theories. Living Rev. Relativ. 2010, 13, 3.

21. Nojiri, S.; Odintsov, S.D. Non-Singular Modified Gravity Unifying Inflation with Late-Time Acceleration and Universality of Viscous Ratio Bound in F(R) Theory. Prog. Theor. Phys. Supp. 2011, 190, 155-178.

22. Capozziello, S.; de Laurentis, M. Extended Theories of Gravity. Phys. Rep. 2011, 509, 167-321.

23. Clifton, T.; Ferreira, P.G.; Padilla, A.; Skordis, C. Modified gravity and cosmology. Phys. Rep. 2012, 513, $1-189$.

24. Capozziello, S.; De Laurentis, M. The dark matter problem from $\mathrm{f}(\mathrm{R})$ gravity viewpoint. Ann. Phys. Berlin 2012, 524, 545-578.

25. Capozziello, S.; Harko, T.; Koivisto, T.; Lobo, F.; Olmo, G. Hybrid Metric-Palatini Gravity. Universe 2015, 1, 199-238.

26. de Martino, I.; De Laurentis, M.; Capozziello, S. Constraining $f(R)$ gravity by the Large Scale Structure. Universe 2015, 1, 123-157

27. Capozziello, S.; de Laurentis, M.; Luongo, O. Connecting early and late universe by f(R) gravity. Int. J. Mod. Phys. D 2015, 24, 1541002.

28. Cai, Y.F.; Capozziello, S.; De Laurentis, M.; Saridakis, E.N. f(T) teleparallel gravity and cosmology. Rep. Prog. Phys. 2016, 79, 106901.

29. Einstein, A. Kosmologische Betrachtungen zur allgemeinen Relativitätstheorie. Das Relativitätsprinzip. Fortschritte der Mathematischen Wissenschaften in Monographien; Vieweg + Teubner Verlag: Wiesbaden, Germany, 1917, pp. 142-152.

30. Islam, J. The cosmological constant and classical tests of general relativity. Phys. Lett. A 1983, 97, $239-241$.

31. Cardona, J.; Tejero, J. Can interplanetary measures bound the cosmological constant? Astrophys. J. 1998, 493, 52-53.

32. Rindler, W. Relativity: Special, General, and Cosmological; Oxford University Press: Oxford, UK, 2001.

33. Kerr, A.; Hauck, J.; Mashhoon, B. Standard clocks, orbital precession and the cosmological constant. Class. Quantum Gravity 2003, 20, 2727-2736.

34. Kraniotis, G.; Whitehouse, S. Compact calculation of the perihelion precession of mercury in general relativity, the cosmological constant and Jacobi's inversion problem. Class. Quantum Gravity 2003, 20, 4817-4835. 
35. Iorio, L. Can solar system observations tell us something about the cosmological constant? Int. J. Mod. Phys. D 2006, 15, 473-475.

36. Jetzer, P.; Sereno, M. Two-body problem with the cosmological constant and observational constraints. Phys. Rev. D 2006, 73, 044015.

37. Kagramanova, V.; Kunz, J.; Lämmerzahl, C. Solar system effects in Schwarzschild-de Sitter space-time. Phys. Lett. B 2006, 634, 465-470.

38. Sereno, M.; Jetzer, P. Solar and stellar system tests of the cosmological constant. Phys. Rev. D 2006, 73, 063004.

39. Adkins, G.; McDonnell, J.; Fell, R. Cosmological perturbations on local systems. Phys. Rev. D 2007, 75, 064011.

40. Adkins, G.; McDonnell, J. Orbital precession due to central-force perturbations. Phys. Rev. D 2007, 75, 082001.

41. Ruggiero, M.L.; Iorio, L. Solar System planetary orbital motions and $\mathrm{f}(\mathrm{R})$ theories of gravity. J. Cosmol. Astropart. Phys. 2007, 1, 010.

42. Sereno, M.; Jetzer, P. Evolution of gravitational orbits in the expanding universe. Phys. Rev. D 2007, 75, 064031.

43. Iorio, L. Solar System Motions and the Cosmological Constant: A New Approach. Adv. Astron. 2008, $2008,268647$.

44. Chashchina, O.I.; Silagadze, Z.K. Remark on orbital precession due to central-force perturbations. Phys. Rev. D 2008, 77, 107502.

45. Iorio, L.; Saridakis, E.N. Solar system constraints on $\mathrm{f}(\mathrm{T})$ gravity. Mon. Not. R. Astron. Soc. 2012, 427, 1555-1561.

46. Arakida, H. Note on the Perihelion/Periastron Advance Due to Cosmological Constant. Int. J. Theor. Phys. 2013, 52, 1408-1414.

47. Xie, Y.; Deng, X.M. f (T) gravity: Effects on astronomical observations and Solar system experiments and upper bounds. Mon. Not. R. Astron. Soc. 2013, 433, 3584-3589.

48. Iorio, L.; Radicella, N.; Ruggiero, M.L. Constraining f(T) gravity in the Solar System. J. Cosmol. Astropart. Phys. 2015, 8, 021.

49. Ovcherenko, S.S.; Silagadze, Z.K. Comment on perihelion advance due to cosmological constant. Ukr. J. Phys. 2016, 61, 342-344.

50. Kottler, F. Über die physikalischen Grundlagen der Einsteinschen Gravitationstheorie. Ann. Phys. Berlin 1918, 361, 401-462.

51. Stuchlík, Z.; Hledík, S. Some properties of the Schwarzschild-de Sitter and Schwarzschild-anti-de Sitter spacetimes. Phys. Rev. D 1999, 60, 044006.

52. Fienga, A.; Laskar, J.; Kuchynka, P.; Manche, H.; Desvignes, G.; Gastineau, M.; Cognard, I.; Theureau, G. The INPOP10a planetary ephemeris and its applications in fundamental physics. Celest. Mech. Dyn. Astron. 2011, 111, 363-385.

53. Hees, A.; Folkner, W.M.; Jacobson, R.A.; Park, R.S. Constraints on modified Newtonian dynamics theories from radio tracking data of the Cassini spacecraft. Phys. Rev. D 2014, 89, 102002.

54. Iorio, L.; Ruggiero, M.L.; Radicella, N.; Saridakis, E.N. Constraining the Schwarzschild-de Sitter solution in models of modified gravity. Phys. Dark Univ. 2016, 13, 111-120.

55. Hees, A.; Lamine, B.; Reynaud, S.; Jaekel, M.T.; Le Poncin-Lafitte, C.; Lainey, V.; Füzfa, A.; Courty, J.M.; Dehant, V.; Wolf, P. Radioscience simulations in general relativity and in alternative theories of gravity. Class. Quantum Gravity 2012, 29, 235027.

56. Pfahl, E.; Loeb, A. Probing the Spacetime around Sagittarius A* with Radio Pulsars. Astrophys. J. 2004, 615, 253-258.

57. Zhang, F.; Lu, Y.; Yu, Q. On the Existence of Pulsars in the Vicinity of the Massive Black Hole in the Galactic Center. Astrophys. J. 2014, 784, 106.

58. Chennamangalam, J.; Lorimer, D.R. The Galactic Centre pulsar population. Mon. Not. R. Astron. Soc. Lett. 2014, 440, L86-L90.

59. Rajwade, K.M.; Lorimer, D.R.; Anderson, L.D. Detecting pulsars in the Galactic Centre. Mon. Not. R. Astron. Soc. 2017, 471, 730-739.

60. Psaltis, D.; Wex, N.; Kramer, M. A Quantitative Test of the No-hair Theorem with Sgr A* Using Stars, Pulsars, and the Event Horizon Telescope. Astrophys. J. 2016, 818, 121.

61. Goddi, C.; Falcke, H.; Kramer, M.; Rezzolla, L.; Brinkerink, C.; Bronzwaer, T.; Davelaar, J.R.J.; Deane, R.; de Laurentis, M.; Desvignes, G.; et al. BlackHoleCam: Fundamental physics of the galactic center. Int. J. Mod. Phys. D 2017, 26, 1730001. 
62. Espriu, D. Pulsar timing arrays and the cosmological constant. AIP Conf. Proc. 2014, 1606, 86-98.

63. Iorio, L. Post-Keplerian perturbations of the orbital time shift in binary pulsars: An analytical formulation with applications to the galactic center. Eur. Phys. J. C 2017, 77, 439.

64. Schücker, T.; Zaimen, N. Cosmological constant and time delay. Astron. Astrophys. 2008, 484, 103-106,

65. Merritt, D.; Alexander, T.; Mikkola, S.; Will, C.M. Stellar dynamics of extreme-mass-ratio inspirals. Phys. Rev. D 2011, 84, 044024.

66. Sadeghian, L.; Will, C.M. Testing the black hole no-hair theorem at the galactic center: Perturbing effects of stars in the surrounding cluster. Class. Quantum Gravity 2011, 28, 225029.

67. Angélil, R.; Saha, P. Clocks around Sgr A*. Mon. Not. R. Astron. Soc. 2014, 444, 3780-3791.

68. Zhang, F.; Iorio, L. On the Newtonian and Spin-induced Perturbations Felt by the Stars Orbiting around the Massive Black Hole in the Galactic Center. Astrophys. J. 2017, 834, 198.

69. Zhang, F.; Saha, P. Probing the spinning of the massive black hole in the Galactic Center via pulsar timing: A Full Relativistic Treatment. Astrophys. J. 2017, 849, 33.

70. De Laurentis, M.; Younsi, Z.; Porth, O.; Mizuno, Y.; Rezzolla, L. Test-particle dynamics in general spherically symmetric black hole spacetimes. arXiv 2017, arXiv:1712.00265.

71. Casotto, S. Position and velocity perturbations in the orbital frame in terms of classical element perturbations. Celest. Mech. Dyn. Astron. 1993, 55, 209-221.

72. Eckart, A.; Hüttemann, A.; Kiefer, C.; Britzen, S.; Zajaček, M.; Lämmerzahl, C.; Stöckler, M.; Valencia-S, M.; Karas, V.; García-Marín, M. The Milky Way's Supermassive Black Hole: How Good a Case Is It? Found. Phys. 2017, 47, 553-624.

73. Gillessen, S.; Plewa, P.M.; Eisenhauer, F.; Sari, R.; Waisberg, I.; Habibi, M.; Pfuhl, O.; George, E.; Dexter, J.; von Fellenberg, S.; et al. An Update on Monitoring Stellar Orbits in the Galactic Center. Astrophys. J. 2017, 837, 30.

74. Hees, A.; Do, T.; Ghez, A.M.; Martinez, G.D.; Naoz, S.; Becklin, E.E.; Boehle, A.; Chappell, S.; Chu, D.; Dehghanfar, A.; et al. Testing General Relativity with Stellar Orbits around the Supermassive Black Hole in Our Galactic Center. Phys. Rev. Lett. 2017, 118, 211101.

75. Lucy, L.B. Mass estimates for visual binaries with incomplete orbits. Astron. Astrophys. 2014, 563, A126.

76. Brumberg, V.A. Essential Relativistic Celestial Mechanics; Taylor \& Francis Group: Boca Raton, FL, USA, 1991.

77. Milani, A.; Nobili, A.; Farinella, P. Non-Gravitational Perturbations and Satellite Geodesy; Taylor \& Francis: Boca Raton, FL, USA, 1987.

78. Soffel, M.H. Relativity in Astrometry, Celestial Mechanics and Geodesy; Springer: Berlin, Germany, 1989.

79. Bertotti, B.; Farinella, P.; Vokrouhlický, D. Physics of the Solar System-Dynamics and Evolution, Space Physics, and Spacetime Structure; Springer: Dordrecht, The Netherlands, 2003. 